\title{
The new electromagnetic tetrads, infinite tetrad nesting and the non trivial emergence of complex numbers in real theories of gravitation
}

\author{
Alcides Garat ${ }^{1}$ \\ 1. Former Professor at Instituto de Física, Facultad de Ciencias, Iguá 4225, \\ esq. Mataojo, Montevideo, Uruguay.
}

(Dated: May 26th, 2012)

How complex numbers get into play in a non trivial way in real theories of gravitation is relevant since in a unified structure they should be able to relate in a natural way with quantum theories. For a long time this issue has been lingering on both relativistic formulations and quantum theories. We will analyze this fundamental subject under the light of new group isomorphism theorems linking local internal groups of transformations and local groups of spacetime transformations. The bridge between these two kinds of transformations are new tetrads introduced previously. It is precisely through these local tetrad structures and constructions that we will provide a non trivial answer to this old question. These new tetrads have two fundamental building components. The skeletons and the gauge vectors. It is these constructive elements that provide the mathematical support that allows to prove group isomorphism theorems. In addition to this, we will prove a unique new property, the infinite tetrad nesting, alternating the nesting with non-Abelian tetrads in the construction of the tetrad gauge vectors. As an application we will demonstrate an alternative proof of a new group isomorphism theorem. 


\section{INTRODUCTION}

The new tetrads introduced in manuscripts ${ }^{1,2}$ have proved very useful in understanding many local geometrical as well as local group properties of gauge theories in four-dimensional curved Lorentzian spacetimes ${ }^{3,4,5}$. There rises the question if it is possible to advance a contribution to the long standing issue of the relationship between general relativistic theories and complex numbers. Stated in an equivalent way, the long standing question about the relationship between general relativistic theories and quantum field theories. Many formulations have been put forward since the beginning of general relativity and quantum mechanics, see for instance the reviews on complex numbers in relativistic theories ${ }^{6,7}$ and all the references therein or the bibliographical literature given in references ${ }^{8,9,10}$. Quantum field theories of gravity using complex associated formulations can be found in the following reviews and all the references therein ${ }^{11-17}$. It is impossible to enumerate all the contributions made in this endeavor. We just get down to the task of presenting our arguments. We work in traditional Abelian and non-Abelian theories in four-dimensional real Lorentzian spacetimes. The complex quantities enter our differential equations through non-Abelian fields locally gauged by non-Abelian Lie groups. It is then fair to ask where exactly complex numbers are going to come into play if our spacetimes are real and the metric tensor is real. The answer and the difference is made through the peculiar structure of our new tetrads. We refer to manuscripts ${ }^{1,2}$ as far as the details of the construction properties of these new tetrads are concerned. These tetrads have two essential elements in their construction. First what we call a skeleton and second a gauge vector, see references ${ }^{1,2,18}$. In section II we will explain these elements in detail. It is through the gauge vectors that complex numbers, complex matrices and also spinors enter in these real formulations. The gauge vectors have real components, because our new tetrads are real and the metric tensor is also real. But the gauge vectors might be constructed using traces of non-trivial products of complex matrices. Matrices associated to representations of Lie groups, for instance. We also engage in another issue that we call tetrad infinite nesting. In the gauge vector structure, we might include contractions with a second set of tetrads. These tetrads will be embedded in the gauge vector construction. These embedded tetrads will need in turn their own local gauge vectors, which in turn might involve a third set of tetrads and so on. Two different sets of tetrads, one electromagnetic Abelian and another $S U(2)$ non-Abelian will be presented in 
section II. We will study the infinite tetrad nesting in section III. In section IV it is our goal as an application to prove that there is an alternative way to establish an isomorphism between $S U(2)$ and the triple tensor product of the one-dimensional LB1 or LB2 which is isomorphic to LB1. We will also make clear what we mean by tensor products of onedimensional groups within the context of tetrad constructions. In section $\mathrm{V}$ we will discuss the non-trivial introduction of complex numbers in real general relativistic field theories. We use a metric with sign conventions -+++ . If $F_{\mu \nu}$ is the electromagnetic field then $f_{\mu \nu}=\left(G^{1 / 2} / c^{2}\right) F_{\mu \nu}$ is the geometrized electromagnetic field and $f_{\mu \nu}^{k}$ are the geometrized Yang-Mills field components, $f_{\mu \nu}^{k}=\left(G^{1 / 2} / c^{2}\right) F_{\mu \nu}^{k}$.

\section{TETRADS IN GAUGE THEORIES OF GRAVITY}

The new tetrads define at every point in spacetime two orthogonal planes or blades. The timelike and one spacelike unit vectors define blade one. The other two unit spacelike vectors define blade two ${ }^{19}$. These tetrads diagonalize locally and covariantly the Yang-Mills stressenergy tensors, see references ${ }^{1,2}$. Blade one and two are local planes of gauge symmetry. Local gauge transformations are isomorphic to tetrad "rotations" both on plane one and two, independently. These local groups of tetrad "rotations" we called LB1 and LB2, see papers $^{1,2}$. LB1 is $S O(1,1)$ plus two different kinds of discrete transformations, one of them is not a Lorentz transformation. LB2 is $S O(2)$. These new tetrads possess two fundamental construction structures, the skeleton and the gauge vector. Our new tetrads have local gauge dependence through the gauge vectors on local Lie groups, see references ${ }^{1,2}$. When we introduce gauge transformations, these are manifested in the gauge vectors and therefore in the tetrad vectors themselves, but not in the tetrad skeletons. Tetrad skeletons are built in a local gauge invariant way. In order to achieve our goals we have to build a tetrad such that $S U(2) \times U(1)$ generated tetrad transformations on blade one, leave the tetrad vectors that generate this blade, on blade one, and similarly for $S U(2) \times U(1)$ generated rotations on blade two. This property is fundamental to ensure the explicit invariance of the metric tensor under $S U(2) \times U(1)$ local gauge transformations. Let us consider for instance the Einstein-Maxwell-Yang-Mills vacuum field equations,

$$
R_{\mu \nu}=T_{\mu \nu}^{(y m)}+T_{\mu \nu}^{(e m)}
$$




$$
\begin{aligned}
f_{; \nu}^{\mu \nu} & =0 \\
* f_{; \nu}^{\mu \nu} & =0 \\
f_{\mid \nu}^{k \mu \nu} & =0 \\
* f_{\mid \nu}^{k \mu \nu} & =0 .
\end{aligned}
$$

The index $k=1 \ldots 3$ is a $S U(2)$ internal index. The dual tensor to the electromagnetic field tensor $f_{\mu \nu}$ is $* f_{\mu \nu}=\frac{1}{2} \epsilon_{\mu \nu \sigma \tau} f^{\sigma \tau}$. The field equations (2-3) suggest the existence of two electromagnetic field potentials, as studied in the first paper "Tetrads in geometrodynamics" ${ }^{1}$, not independent from each other, but due to the symmetry of the equations, available for our construction. $A^{\mu}$ and $* A^{\mu}$ are the two electromagnetic potentials. $* A^{\mu}$ is therefore a name, we are not using the Hodge map at all in this case. We will see that we can write $f_{\mu \nu}=A_{\nu ; \mu}-A_{\mu ; \nu}$ and $* f_{\mu \nu}=* A_{\nu ; \mu}-* A_{\mu ; \nu}$. Similar for the two NonAbelian equations (4-5). The Non-Abelian potential $A^{k \mu}$ is available for our construction as well. By the symbol | we mean gauge covariant derivatives. For instance, the gauge covariant derivatives of the three field strength internal components $f_{k \mu \nu \mid \rho}=f_{k \mu \nu ; \rho}+g \epsilon_{k l p} A_{l \rho} f_{p \mu \nu}$, where $\epsilon_{k l p}$ is the completely skew-symmetric tensor in three dimensions with $\epsilon_{123}=1$, and $g$ is the coupling constant. The symbol ";" stands for the usual covariant derivative associated with the metric tensor $g_{\mu \nu}$. We will describe briefly the tetrad construction process in both the electromagnetic case $U(1)$ and the non-Abelian case $S U(2) \times U(1)$. In the Abelian electromagnetic case a local duality transformation enabled us to introduce an extremal field,

$$
\xi_{\mu \nu}=e^{-* \alpha} f_{\mu \nu}=\cos \alpha f_{\mu \nu}-\sin \alpha * f_{\mu \nu}
$$

The local scalar $\alpha$ is defined by imposing the condition,

$$
\xi_{\mu \nu} * \xi^{\mu \nu}=0
$$

such that the expression for $\alpha$ turns out to be $\tan (2 \alpha)=-f_{\mu \nu} * f^{\mu \nu} / f_{\lambda \rho} f^{\lambda \rho}$. The identity,

$$
A_{\mu \alpha} B^{\nu \alpha}-* B_{\mu \alpha} * A^{\nu \alpha}=\frac{1}{2} \delta_{\mu}{ }^{\nu} A_{\alpha \beta} B^{\alpha \beta}
$$

valid for every pair of antisymmetric tensors in a four-dimensional Lorentzian spacetimes ${ }^{18}$, when applied to the case $A_{\mu \alpha}=\xi_{\mu \alpha}$ and $B^{\nu \alpha}=* \xi^{\nu \alpha}$ yields the equivalent extremal condition, 


$$
\xi_{\alpha \mu} * \xi^{\mu \nu}=0
$$

Thus, we can proceed to introduce the new tetrad,

$$
\begin{aligned}
U^{\alpha} & =\xi^{\alpha \lambda} \xi_{\rho \lambda} A^{\rho} /\left(\sqrt{-Q / 2} \sqrt{A_{\mu} \xi^{\mu \sigma} \xi_{\nu \sigma} A^{\nu}}\right) \\
V^{\alpha} & =\xi^{\alpha \lambda} A_{\lambda} /\left(\sqrt{A_{\mu} \xi^{\mu \sigma} \xi_{\nu \sigma} A^{\nu}}\right) \\
Z^{\alpha} & =* \xi^{\alpha \lambda} * A_{\lambda} /\left(\sqrt{* A_{\mu} * \xi^{\mu \sigma} * \xi_{\nu \sigma} * A^{\nu}}\right) \\
W^{\alpha} & =* \xi^{\alpha \lambda} * \xi_{\rho \lambda} * A^{\rho} /\left(\sqrt{-Q / 2} \sqrt{* A_{\mu} * \xi^{\mu \sigma} * \xi_{\nu \sigma} * A^{\nu}}\right) .
\end{aligned}
$$

Where $Q=\xi_{\mu \nu} \xi^{\mu \nu}=-\sqrt{T_{\mu \nu} T^{\mu \nu}}$ according to equations (39) in ${ }^{18}$. $Q$ is assumed not to be zero, because we are dealing with non-null electromagnetic fields. Let us introduce some names. The tetrad vectors have two essential components as stated above. For instance in vector $U^{\alpha}$ there are two main structures. First, the skeleton, in this case $\xi^{\alpha \lambda} \xi_{\rho \lambda}$, and second, the gauge vector $A^{\rho}$. The gauge vectors it was proved in manuscript ${ }^{1}$ could be anything $X^{\rho}$ that does not make the tetrad vectors trivial, it is a gauge choice. The four vectors (10-13) have the following algebraic properties,

$$
-U^{\alpha} U_{\alpha}=V^{\alpha} V_{\alpha}=Z^{\alpha} Z_{\alpha}=W^{\alpha} W_{\alpha}=1
$$

Using equations (8-9) it is simple to prove that (10-13) are orthonormal. The duality rotation given by equation (6) enables us to express the stress-energy tensor in terms of the extremal field,

$$
T_{\mu \nu}=\xi_{\mu \lambda} \xi_{\nu}^{\lambda}+* \xi_{\mu \lambda} * \xi_{\nu}^{\lambda}
$$

With all these elements it becomes trivial to prove that the tetrad (10-13) diagonalizes locally and covariantly the stress-energy tensor (15), see reference ${ }^{1}$. We observe that the extremal field $\xi_{\mu \nu}$ is a local gauge invariant under electromagnetic $U(1)$ gauge transformations. The only dependence on electromagnetic gauge of the tetrad (10-13) is through both the gauge vectors, $A^{\mu}$ and $* A^{\mu}$. Finally, let us remember that in the electromagnetic Abelian case, the Maxwell equations, 


$$
\begin{gathered}
f_{; \nu}^{\mu \nu}=0 \\
* f_{; \nu}^{\mu \nu}=0,
\end{gathered}
$$

are informing us that two potential vector fields $A_{\nu}$ and $* A_{\nu}$ exist $^{20}$,

$$
\begin{gathered}
f_{\mu \nu}=A_{\nu ; \mu}-A_{\mu ; \nu} \\
* f_{\mu \nu}=* A_{\nu ; \mu}-* A_{\mu ; \nu} .
\end{gathered}
$$

The symbol ";, stands for covariant derivative with respect to the metric tensor $g_{\mu \nu}$. In the non-Abelian case we make use of a generalized local duality rotation, this time in isospace. But we first introduce the following antisymmetric auxiliary field,

$$
\varepsilon_{\mu \nu}=\operatorname{Tr}\left[\vec{n} \cdot f_{\mu \nu}-\vec{l} \cdot * f_{\mu \nu}\right]
$$

where $f_{\mu \nu}=f_{\mu \nu}^{a} \sigma^{a}$ is the non-Abelian field strength, $\vec{n}=n^{a} \sigma^{a}$ and $\vec{l}=l^{a} \sigma^{a}$ are vectors in isospace. The $\cdot$ means product in isospace. $\sigma^{a}$ are the pauli matrices and the summation convention is applied on the internal index $a=1 \ldots 3$. The vector components are defined as,

$$
\begin{aligned}
& \vec{n}=\left(\cos \theta_{1}, \cos \theta_{2}, \cos \theta_{3}\right) \\
& \vec{l}=\left(\cos \beta_{1}, \cos \beta_{2}, \cos \beta_{3}\right),
\end{aligned}
$$

where all the six isoangles are local scalars that satisfy,

$$
\begin{aligned}
& \Sigma_{a=1}^{3} \cos ^{2} \theta_{a}=1 \\
& \Sigma_{a=1}^{3} \cos ^{2} \beta_{a}=1 .
\end{aligned}
$$

In isospace $\vec{n}=n^{a} \sigma^{a}$ transforms under a local $S U(2)$ gauge transformation $S$, as $S^{-1} \vec{n} S$, see chapter III in ${ }^{21}$ and also reference ${ }^{22}$, and similar for $\vec{l}=l^{a} \sigma^{a}$. The tensor $f_{\mu \nu}=f_{\mu \nu}^{a} \sigma^{a}$ transforms as $f_{\mu \nu} \rightarrow S^{-1} f_{\mu \nu} S$. Therefore $\varepsilon_{\mu \nu}$ is manifestly gauge invariant. We can see from (19-20) and (21-22) that only four of the six angles in isospace are independent. Next, we present the extremal field by carrying out one more duality transformation, 


$$
\epsilon_{\mu \nu}=\cos \alpha_{d} \varepsilon_{\mu \nu}-\sin \alpha_{d} * \varepsilon_{\mu \nu}
$$

such that the complexion $\alpha_{d}$ is defined by the standard local condition $\epsilon_{\mu \nu} * \epsilon^{\mu \nu}=0$, see reference $^{1,2}$,

$$
\tan \left(2 \alpha_{d}\right)=-\varepsilon_{\mu \nu} * \varepsilon^{\mu \nu} / \varepsilon_{\lambda \rho} \varepsilon^{\lambda \rho}
$$

When the identity (8) is used in the case $A_{\mu \alpha}=\epsilon_{\mu \alpha}$ and $B^{\nu \alpha}=* \epsilon^{\nu \alpha}$, it yields the equivalent extremal condition,

$$
\epsilon_{\alpha \mu} * \epsilon^{\mu \nu}=0
$$

It is this extremal field $\epsilon_{\mu \nu}$ that we use in order to construct the orthogonal tetrad,

$$
\begin{aligned}
& S_{1}^{\mu}=\epsilon^{\mu \lambda} \epsilon_{\rho \lambda} X^{\rho} /\left(\sqrt{-Q_{y m} / 2} \sqrt{X_{\tau} \epsilon^{\tau \sigma} \epsilon_{\nu \sigma} X^{\nu}}\right) \\
& S_{2}^{\mu}=\epsilon^{\mu \lambda} X_{\lambda} /\left(\sqrt{X_{\tau} \epsilon^{\tau \sigma} \epsilon_{\nu \sigma} X^{\nu}}\right) \\
& S_{3}^{\mu}=* \epsilon^{\mu \lambda} Y_{\lambda} /\left(\sqrt{* Y_{\tau} * \epsilon^{\tau \sigma} * \epsilon_{\nu \sigma} * Y^{\nu}}\right) \\
& S_{4}^{\mu}=* \epsilon^{\mu \lambda} * \epsilon_{\rho \lambda} Y^{\rho} /\left(\sqrt{-Q_{y m} / 2} \sqrt{* Y_{\tau} * \epsilon^{\tau \sigma} * \epsilon_{\nu \sigma} * Y^{\nu}}\right),
\end{aligned}
$$

where $Q_{y m}=\epsilon_{\mu \nu} \epsilon^{\mu \nu}$ is assumed not to be zero. Tetrad vectors (26-27) define blade one , and (28-29) blade two, at every point in spacetime. We are going to call as stated previously, for instance $\epsilon^{\mu \lambda} \epsilon_{\rho \lambda}$ the skeleton of the tetrad vector $S_{1}^{\mu}$, and $X^{\rho}$ the gauge vector. In the case of $S_{3}^{\mu}$, the skeleton will be $* \epsilon^{\mu \lambda}$, and $Y_{\lambda}$ will be the gauge vector. It is evident by construction that skeletons are gauge invariant. This property guarantees that the vectors under local $U(1)$ or $S U(2)$ gauge transformations are not going to leave their original planes or blades, keeping therefore the metric tensor explicitly invariant, see references ${ }^{1,2}$.

\section{INFINITE TETRAD NESTING}

In order to appreciate this new tetrad local property of infinite tetrad nesting, we have to build special kinds of tetrad gauge vectors. Let us make a first choice and start with the local gauge vector introduced in manuscript ${ }^{2}$, 


$$
X^{\sigma}=Y^{\sigma}=\operatorname{Tr}\left[\Sigma^{\alpha \beta} E_{\alpha}^{\rho} E_{\beta}^{\lambda} * \xi_{\rho}^{\sigma} * \xi_{\lambda \tau} A^{\tau}\right] .
$$

The antisymmetric object $\Sigma^{\alpha \beta}$ is defined as $\Sigma^{\alpha \beta}=\sigma_{+}^{\alpha} \sigma_{-}^{\beta}-\sigma_{+}^{\beta} \sigma_{-}^{\alpha}{ }^{23-28}$. The object $\sigma_{ \pm}^{\alpha}$ arises when building the Weyl representation for left handed and right handed spinors. According to ${ }^{26}$, it is defined as $\sigma_{ \pm}^{\alpha}=\left(\mathbf{1}, \pm \sigma^{\mathbf{i}}\right)$, where $\sigma^{i}$ are the Pauli matrices for $i=$ $1 \cdots 3$. This object $\Sigma^{\alpha \beta}$ was built and studied in the second appendix of reference ${ }^{2}$. The potential $A^{\tau}$ is the non-Abelian $S U(2)$ gauge potential. $E_{\alpha}{ }^{\rho}$ are tetrad vectors that transform from a locally inertial coordinate system, into a general curvilinear coordinate system. In this particular section, greek indices $\alpha, \beta, \delta, \epsilon, \gamma$, and $\kappa$, are reserved for locally inertial coordinate systems. We can make a particular explicit choice for these tetrads $E_{\alpha}{ }^{\rho}$. We can choose the tetrad vectors we already know from paper ${ }^{1}$ and section II, for electromagnetic fields in curved space-times. Following the same notation as in $^{1}$ and section II, we call $E_{o}{ }^{\rho}=U^{\rho},{E_{1}}^{\rho}=V^{\rho},{E_{2}}^{\rho}=Z^{\rho},{E_{3}}^{\rho}=W^{\rho}$. With the additional observation that for the electromagnetic tetrads we are not going immediately to choose gauge vectors, then obtaining,

$$
\begin{aligned}
& E_{o}^{\mu}=\xi^{\mu \lambda} \xi_{\rho \lambda} X^{\rho} /\left(\sqrt{-Q / 2} \sqrt{X_{\tau} \xi^{\tau \sigma} \xi_{\nu \sigma} X^{\nu}}\right) \\
& E_{1}^{\mu}=\xi^{\mu \lambda} X_{\lambda} /\left(\sqrt{X_{\tau} \xi^{\tau \sigma} \xi_{\nu \sigma} X^{\nu}}\right) \\
& E_{2}^{\mu}=* \xi^{\mu \lambda} * Y_{\lambda} /\left(\sqrt{* Y_{\tau} * \xi^{\tau \sigma} * \xi_{\nu \sigma} * Y^{\nu}}\right) \\
& E_{3}^{\mu}=* \xi^{\mu \lambda} * \xi_{\rho \lambda} * Y^{\rho} /\left(\sqrt{-Q / 2} \sqrt{* Y_{\tau} * \xi^{\tau \sigma} * \xi_{\nu \sigma} * Y^{\nu}}\right) .
\end{aligned}
$$

Therefore, we are making use of the already defined electromagnetic tetrads built for space-times where electromagnetic fields are present, in order to allow for the use of the object $\Sigma^{\alpha \beta}$ which is key in our construction. The key lies in the translating quality of this object between $S U(2)$ local gauge transformations $S$ and local Lorentz transformations $\Lambda$, $S^{-1} \Sigma^{\alpha \beta} S=\Lambda_{\gamma}^{\alpha} \Lambda_{\delta}^{\beta} \Sigma^{\gamma \delta}$, see reference ${ }^{2}$. We would like to consider one more property of these chosen vector fields $X^{\rho}$ and $Y^{\rho}$. The structure $E_{\alpha}^{[\rho} E_{\beta}^{\lambda]} * \xi_{\rho \sigma} * \xi_{\lambda \tau}$ is invariant under $U(1)$ local gauge transformations. Essentially, because of the electromagnetic extremal field property ${ }^{1,18}, \xi_{\mu \sigma} * \xi^{\mu \tau}=0$. Leaving thus in the contraction with $* \xi_{\rho \sigma} * \xi_{\lambda \tau}$, and because of property (9), only the antisymmetric object ${E_{2}}^{[\rho} E_{3}{ }^{\lambda]}$, which is locally $U(1)$ gauge 
invariant. Let us remember that the object $\Sigma^{\alpha \beta}$ is antisymmetric and contracted with the electromagnetic tetrads as $\Sigma^{\alpha \beta} E_{\alpha}{ }^{\rho} E_{\beta}{ }^{\lambda}$ inside the local gauge vector (30). Let us now make a second choice for local gauge vectors,

$$
X^{\sigma}=Y^{\sigma}=\operatorname{Tr}\left[\Sigma^{\alpha \beta} S_{\alpha}{ }^{\rho} S_{\beta}{ }^{\lambda} * \epsilon_{\rho}^{\sigma} * \epsilon_{\lambda \tau} A^{\tau}\right]
$$

The analysis of the local gauge vector (35) is similar to that of the local gauge vector (30). We can just follow the explanations above for the electromagnetic $U(1)$ case. The tetrads $S_{\alpha}{ }^{\rho}$ are now the four vectors (26-29). We can also point out that the local covariant structure $S_{\alpha}^{[\rho} S_{\beta}^{\lambda]} * \epsilon_{\rho \sigma} * \epsilon_{\lambda \tau}$ is now invariant under $S U(2)$ local gauge transformations, for the same reasons expressed above for the $U(1)$ case, see reference ${ }^{2}$. Once we have presented all these elements, we can state the following. Let us gauge our electromagnetic tetrads (31-34) with the local gauge vector (35). Then, inside this local gauge vector we have the tetrads (26-29). Next, let us proceed to gauge these (26-29) tetrads with the local gauge vector (30). Since we are nesting alternatingly in the local gauge vectors, the local $S U(2)$ tetrads (2629) and the local electromagnetic tetrads (31-34), starting with the electromagnetic tetrad, for example, we can produce infinite local tetrad nesting. Unless we interrupt the nesting process gauging locally with $A^{\mu}$ and $* A^{\mu}$, for instance. The point is that we have this new unique property of infinite tetrad nesting, telling us about the possibilities associated with tetrad and metric construction in four-dimensional Lorentzian spacetimes and the system of differential equations (1-5). Let us call the first original electromagnetic tetrad, the zero-th nesting level. Then, the non-Abelian tetrad in the electromagnetic gauge vector we call the first nesting level, and so on. Let us suppose that at every point in spacetime we gauge the n-th level with $A^{\mu}$ and $* A^{\mu}$, therefore interrupting the nesting process. We have then at every point a structure with n-th nesting level tetrads. There are many possibilities. We could build eventually by similar algorithms to the ones depicted in papers ${ }^{1,2}, S U(N)$ tetrads with the corresponding $S U(N)$ skeletons and gauge vectors and the corresponding associated system of differential equations. Then, we could embed at the n-th nesting level gauge vectors, these $S U(n)$ tetrads, therefore not alternating but always using a different one at every nesting level. We leave as an open subject, or open question if these structures can be used in transfinite G. Cantor mathematics ${ }^{29,30}$. Open issues created by this infinite tetrad nesting properties. 


\section{APPLICATION: NEW FORM OF ISOMORPHISM THEOREMS}

It is our goal in this section to prove that there is an alternative way to establish an isomorphism between $S U(2)$ and the triple tensor product of the one-dimensional LB1 or LB2 which is isomorphic to LB1. On a local plane one or two spanned by just two of our tetrad vectors with a single and unique extremal field-gauge vector structure, the tensor product of one-dimensional groups like LB1 or LB2 would just be one-dimensional. However this is not the case we are discussing in this manuscript and we will clarify this situation during the analysis. The proof runs along the lines already formulated in detail in manuscript ${ }^{2}$. Therefore we will concentrate on the tetrad nesting and the gauge vectors associated to the tetrad nesting levels. Let us start with the electromagnetic tetrad $E_{\alpha}{ }^{\mu}$ presented in equations (31-34). Let us next choose as gauge vectors $X_{1}^{\sigma}=Y_{1}^{\sigma}=\operatorname{Tr}\left[\Sigma^{\alpha \beta} S_{\alpha}{ }^{\rho} S_{\beta}{ }^{\lambda} * \epsilon_{\rho}^{\sigma} * \epsilon_{\lambda \tau} A^{\tau}\right]$ where the $A^{\tau}$ is the $S U(2)$ connection. Finally, let us gauge the $S U(2)$ tetrads $S_{\alpha}{ }^{\mu}$ nested at first nesting level with $X_{2}^{\sigma}=A^{\sigma}$ and $Y_{2}^{\sigma}=* A^{\sigma}$ where these connections are the electromagnetic potentials. This second gauge choice is realized at second nesting level. We stop the nesting there, since with our second gauge choices there are no more nested tetrads. If we now proceed to consider local $S U(2)$ gauge transformations of the connection at first level, we will find that the result are two composed local transformations as studied in absolute detail in paper ${ }^{2}$. First, a local spatial rotation of the tetrads $S_{\alpha}{ }^{\mu}$ inside the gauge vector at first nesting level. Second, a transformation LB1 on the local plane one or LB2 on the local plane two determined by the level zeroth electromagnetic $E_{\alpha}{ }^{\mu}$ tetrad vectors. We now separately consider two more different spatially and locally rotated electromagnetic tetrads $\tilde{E}_{\alpha}^{\mu}$ and $\bar{E}_{\alpha}^{\mu}$ with exactly the same first and second level gauge vectors as the original $E_{\alpha}{ }^{\mu}$. Subsequently we proceed to consider local $S U(2)$ gauge transformations in these additional tetrads $\tilde{E}_{\alpha}{ }^{\mu}$ and $\bar{E}_{\alpha}^{\mu}$. We are considering three copies of the same spacetime, and a different

tetrad at the same point in each spacetime copy, that is $E_{\alpha}{ }^{\mu}, \tilde{E}_{\alpha}{ }^{\mu}$ and $\bar{E}_{\alpha}^{\mu}$. These three tetrads have a mutually similar extremal field-gauge vector structure. They are normalized and the same choice of gauge vector has been made. But they are not the same. They could be Lorentz transformed into each other, under non-trivial Lorentz spatial rotations, for example. We know from manuscript ${ }^{2}$ that a local Lorentz transformation of a tetrad with an extremal field-gauge vector structure transforms into another tetrad with a similar extremal field-gauge vector structure, not the same local extremal field. Therefore, we are considering 
three local tetrads at the same spacetime point which are not the same for different copies. The local planes one and two will be tilted with respect to each other. This is what we mean by three LB1 or LB2 groups under tensor product. Now, from a practical point of view what we also mean by these ideas is that we are able to reconstruct the original local $S U(2)$ gauge transformations by knowing for example, the boosts in the LB1 case, or the spatial rotations in the LB2 case, for the tetrad local transformations at the point under consideration. By knowing the local Lorentz transformation values for the three copies, and given all the fields, specially the three electromagnetic tetrads at the same point, we can reconstruct the original local $S U(2)$ gauge transformation that gave rise either to three local LB1 transformations or independently to three local LB2 spatial transformations. Because the isomorphism theorems are proved by following exactly the same analytic steps as in papers $^{1,2}$, representing local isomorphisms between either three LB1 groups and the local group of $S U(2)$ gauge transformations or independently three LB2 groups and the local group of $S U(2)$ gauge transformations. We used the nesting techniques to invert the tetrad nesting. In manuscript ${ }^{2}$ we consider three zeroth level $S U(2)$ tetrads in three copies of the same spacetime, with a first nesting given by electromagnetic tetrads. In this proof we did the opposite, we considered three zeroth level electromagnetic tetrads in three copies of the same spacetime, with a first nesting given by the $S U(2)$ tetrads. If we follow the same steps as in manuscript ${ }^{2}$ the theorems are proved and stated exactly in the same fashion,

Theorem 1 The mapping between the local gauge group SU(2) of transformations and the tensor product of the three local groups of LB1 tetrad transformations is isomorphic.

We can also state,

Theorem 2 The mapping between the local gauge group SU(2) of transformations and the tensor product of the three local groups of LB2 tetrad transformations is isomorphic.

\section{CONCLUSIONS: COMPLEX NUMBERS IN GEOMETRODYNAMICS}

It is a historical issue that has prompted the thoughts of thousands of experts. How to relate real theories of a general relativistic nature with quantum field theories of a complex nature. We provide an answer. Through the gauge vectors. The skeletons can also be associated to complex structures like in the antisymmetric auxiliary field (18). However the 
skeletons must be locally gauge invariant if we wish to keep the local tetrad vectors in the same blades where they started under local gauge transformations, in order for the metric tensor to remain invariant. The gauge vectors are gauge per se, and contain gauge in their own structure. In the pure electromagnetic case we can just gauge with the two potentials, $X^{\mu}=A^{\mu}$ and $Y^{\mu}=* A^{\mu}$, compare tetrads (10-13) with (31-34). In the non-Abelian case we need more sofisticated constructions. As an example we have the $S U(2)$ local gauge vectors (30) or (35). Let us remember that the local gauge vectors (30) and (35) are invariant under local $U(1)$ electromagnetic gauge transformations. These constructions involve traces of products of matrices, included in the non-Abelian potential $A^{\tau}$ and the object $\Sigma^{\alpha \beta}$ that involves, in turn, products of pauli matrices. This object was analyzed in detail in the second appendix of reference ${ }^{2}$. These traces of products of complex matrices give the gauge vectors a non-trivial complex nature. Simultaneously, these gauge vectors are real, essentially because of the Hermitian nature of the matrices involved. Under the light of the theorems proved in manuscripts ${ }^{1,2}$ we can conjecture on the possibility for microparticles to have associated spacetimes. Because all the symmetries associated to different standard model multiplets can be realized in four-dimensional curved spacetimes. Brought into evidence through our new tetrads. When we perform a local gauge transformation, the local tetrads get transformed under LB1 or LB2 into new tetrad fields that happen to lie on the same original local planes one and two. The local planes of gauge symmetry. It is then possible that multiplets can be interpreted as "tetrad states" of the gravitational field, therefore establishing a very concrete link between general relativistic real formulations and quantum field theories. The standard model has been designed on the pillar of gauge invariance. Finding this relation amounts to finding the relationship to the gravitational field. The local tetrads will provide the metric tensor. However, the relevant point is that now we know that the "link" between internal structures and spacetime structures is bridged by tetrads, and local gauge transformations are isomorphic to local tetrad transformations that explicitly leave the metric tensor invariant, see the theorems in papers $^{1,2}$. Let us consider then a new problem involving spinors and a new choice for the two gauge vectors $Y^{\mu}=X^{\mu}=E_{\alpha}^{\mu} \bar{\psi} \sigma^{\alpha} \psi$. It is natural to think of this choice in many problems involving spinors $\psi$. A gauge transformation of the spinors would be translated into a locally inertial transformation of the electromagnetic tetrad $E_{\alpha}^{\mu}$. $Y^{\mu}=X^{\mu} \rightarrow \Lambda_{\beta}^{\alpha} E_{\alpha}^{\mu} \bar{\psi} \sigma^{\beta} \psi$. This would directly imply that when the states represented by the spinor field, locally transform, the 
tetrads rotate in blades one and two. This is then, a direct and explicit "link" between the spinor states and the states of the gravitational field. A direct non-trivial and explicit "link" between the complex numbers and the real gravitational field. As an application in section IV we found an alternative way to establish an isomorphism between $S U(2)$ and the triple tensor product of the one-dimensional LB1 group or the one-dimensional LB2, which is isomorphic to LB1. We quote from" "it would hardly be possible for me to make without constraint the least step forwards in the theory of aggregates", and although "I was led to them many years ago, without arriving at a clear consciousness that I possessed in them concrete numbers of real significance, yet I was logically forced, almost against my will, because in opposition to traditions which had become valued by me in the course of scientific researches extending over many years, to the thought of considering the infinitely great, not merely in the form of the unlimitedly increasing, and in the form, closely connected with this, of convergent infinite series, but also to fix it mathematically by numbers in the definite form of a completed infinite. I do not believe, then, that any reasons can be urged against it which I am unable to combat".

\section{REFERENCES}

${ }^{1}$ A. Garat, J. Math. Phys. 46, 102502 (2005). A. Garat, Erratum: Tetrads in geometrodynamics, J. Math. Phys. 55, 019902 (2014).

${ }^{2}$ A. Garat, Gravitation and Cosmology, 2014 Vol. 20 No. 1, pp. 116-126. Pleiades Publishing Ltd. arXiv:gr-qc/0602049.

${ }^{3}$ A. Garat, Euler observers in geometrodynamics, Int. J. Geom. Meth. Mod. Phys., Vol. 11 (2014), 1450060. arXiv:gr-qc/1306.4005

${ }^{4}$ A. Garat, The Monopole and the Coulomb field as duals within the unifying ReissnerNordström geometry, Communications in Theoretical Phys. 61, No. 6, 699 (2014). A. Garat, arXiv:1306.5784.

${ }^{5}$ A. Garat, https://zenodo.org/record/7587.

${ }^{6} \mathrm{G}$. Esposito, Complex geometry of nature and general relativity, 1999. arXiv:grqc/9911051.

${ }^{7}$ G. Esposito, Int. J. Geom. Meth. Mod. Phys., Vol. 2 675-731 (2005) From spinor geometry to complex general relativity. arXiv:hep-th/0504089/2005. 
${ }^{8}$ R. Penrose and W. Rindler, Spinors and spacetime, Vol. I: Two-spinor calculus and relativistic fields, (Cambridge, Cambridge University Press, 1984).

${ }^{9}$ R. Penrose and W. Rindler, Spinors and spacetime, Vol. II: Spinor and twistor methods in spacetime geometry, (Cambridge, Cambridge University Press, 1986).

${ }^{10}$ J. B. Barbour, Time and Complex Numbers in Canonical Quantum Gravity, Phys. Rev. D 47, $5422(1993)$.

${ }^{11}$ J. Pullin, Canonical Quantization of General Relativity: The Last 18 Years in a Nutshell (2002). arXiv:gr-qc/0209008.

${ }^{12}$ J. W. Moffat, Noncommutative Quantum Gravity. arXiv:hep-th/0007181/2000.

${ }^{13}$ B. Schulz, Review on the Quantization of Gravity. arXiv:1409.7977/2014.

${ }^{14}$ H. Sahlmann, Loop Quantum Gravity: a short review. arXiv:gr-qc/1001.4188/2011.

${ }^{15}$ C. Rovelli, Living Rev. Relativity, Loop Quantum Gravity (1998). http://www.livingreviews.org/lrr-1998-1.

${ }^{16} \mathrm{~T}$. Thiemann, Introduction to Modern Canonical Quantum General Relativity (Cambridge Monographs on Mathematical Physics, Cambridge University Press, Cambridge, 2007).

${ }^{17}$ T. Damour, General Relativity Today. arXiv:gr-qc/0704.0754/2007.

${ }^{18}$ C. Misner and J. A. Wheeler, Annals of Physics 2, 525 (1957).

${ }^{19}$ J. A. Schouten, Ricci Calculus: An Introduction to Tensor Calculus and Its Geometrical Applications (Springer, Berlin, 1954).

${ }^{20}$ N. Cabibbo and E. Ferrari, Nuovo Cim. 23, 1147 (1962).

${ }^{21}$ Y. Choquet-Bruhat and C. DeWitt-Morette, Analysis, Manifolds and Physics (Elsevier Science Publishers B.V., 1987).

${ }^{22}$ W. Greiner and B. Mueller, Quantum Mechanics, Symmetries (Springer Verlag Gmbh, 1989).

${ }^{23}$ M. Carmeli, Classical Fields: General Relativity and Gauge Theory (J. Wiley \& Sons, New York, 1982).

${ }^{24} \mathrm{R}$. Penrose and W. Rindler, Spinors and Spacetime: Volume I (Cambridge University Press, 1984).

${ }^{25}$ M. Kaku, Quantum Field Theory: A Modern Introduction (Oxford University Press, 1993). ${ }^{26}$ L. Álvarez-Gaumé and M. A. Vázquez-Mozo, Introductory Lectures on Quantum Field Theory (arXiv:hep-th/0510040).

${ }^{27}$ M. Nakahara, Geometry, Topology and Physics (Institute of Physics Publishing, 1990). 
${ }^{28}$ M.E. Peskin and D.V. Schroeder, An Introduction to Quantum Field Theory (Perseus Books Publishing L.L.C., 1995).

${ }^{29}$ A. N. Kolmogorov and S. V. Fomin, Elements of the Theory of Functions and Functional Analysis (Dover Books on Mathematics, 1961).

${ }^{30} \mathrm{G}$. Cantor, Contribution to the Founding of the Theory of Transfinite Numbers (Dover Publications, Philip Jourdain Editor, New York, 1955). 